\title{
A novel non-data-aided frequency estimation algorithm for M-PSK signals
}

\author{
Tian Qinghua ${ }^{1, a}$, Man Xin ${ }^{2, b}$ and Zeng Sheng ${ }^{3, c}$ \\ ${ }^{1}$ Huaihua Radio and TV University, Huaihua 418000, P. R. China \\ ${ }^{2}$ National University of Defense Technology, Changsha 410073, P. R. China \\ ${ }^{3}$ Huazhong University of Science and Technology, Wuhan 430074, P. R. China \\ a33632353@qq.com, bmanxin09@163.com, 'dan.edi.zs@gmail.com
}

Keywords: carrier frequency estimation; MPSK; non-data-aided (NDA); step factor

\begin{abstract}
This paper deals with the problem of carrier frequency estimation for MPSK symbols. We present a novel non-data-aided method by introducing a step factor into an earlier estimation algorithm to reduce the computational complexity with little loss of the estimation accuracy. We also make some comparisons with earlier algorithms. From the results of simulations, we make the conclusion that the new method can separate the estimation range and the estimation accuracy, and avoid the weakness of reaching large estimation range at the expense of estimation accuracy.
\end{abstract}

\section{Introduction}

In digital communications, the purpose of any receiver is to decrease as much as possible the number of wrong decisions made on the transmitted data. In order to detect the transmitted bits correctly, a receiver has firstly to estimate a number of synchronization parameters. This paper deals with the problem of carrier frequency offset estimation.

Most of the classical synchronizers are derived from the maximum-likelihood (ML) criterion and usually work in data-aided (DA) or non-data-aided (NDA) modes [1-3]. DA synchronizers lead to expenses in terms of bandwidth and power, which can be avoided for NDA synchronizer. This paper will introduce a NDA method to estimate the carrier frequency offset.

MPSK modulation has been widely used in communication field and there are many synchronization algorithms for MPSK systems. The Fitz algorithm [4] and The Luise\&Reggianini (L\&R) [5] algorithm are important DA carrier frequency estimation methods suitable for MPSK signals. But they possess the character of reaching high estimation accuracy with very small estimation range or reaching large range with low accuracy. Although the Mengali\&Morelli (M\&M) [6] algorithm resolves this problem effectively, it is too complex to implement.

We propose a new NDA method based on the M\&M algorithm for MPSK systems, which has some advantages over the aforementioned methods. Firstly, it introduces a step factor into the M\&M algorithm to reduce the computational complexity with variable acquisition range and little loss of estimation accuracy. Secondly, with a given estimation accuracy, its acquisition range is larger than that of the Fitz algorithm as it separates the estimation range and the estimation accuracy, and avoids the weakness of reaching high estimation accuracy at the expense of estimation range. In a word, the proposed method is more flexible and applicable than both the M\&M and the Fitz algorithms.

\section{System Model}

In Fig. 1 the receiver block diagram is reported. Assuming negligible filter distortion, sampling at the symbol rate, and perfect timing recovery in the digital receiver, the complex samples at the output of the matched filter can be expressed as:

$$
x(k)=c_{k} e^{j(2 \pi \Delta f k T+\theta)}+n_{k}
$$




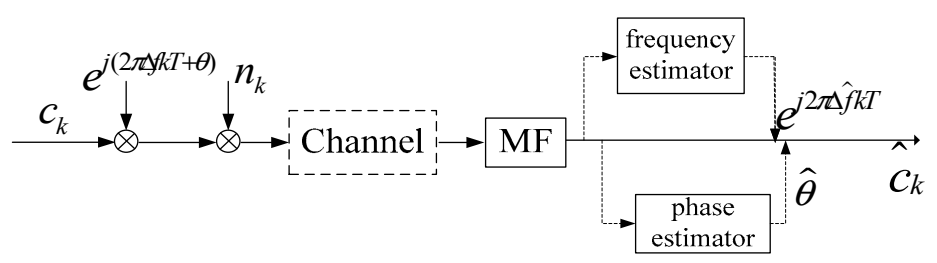

Fig. 1 the receiver block diagram

where $c_{k}$ is unit-amplitude symbol, $n_{k}$ is the complex additional gaussian white noise with

two-sided power spectral density $N_{0} / 2 . \Delta f T$ represents the residual carrier frequency offset, due to Doppler shift and local oscillator mismatch, normalized to the symbol rate and $\theta$ accounts for the phase offset between local oscillators within the transmitter and the receiver. The normalized carrier frequency offset $\Delta f T$ and the phase offset $\theta$ are assumed to be deterministic and constant during the transmission. This paper focuses on the frequency estimator in Fig.1.

From Eq.1, we can see that $x(k)$ depends on $c_{k}$ which is exactly known by the receivers in DA mode such as the M\&M algorithm. The method removes the dependence on $c_{k}$ by multiplying $x(k)$ by $c_{k}^{*}$, which gets

$$
z(k)=e^{j(2 \pi \Delta f k T+\theta)}+n_{k} c_{k}^{*}
$$

where $c_{k}^{*}$ is the conjugation of $c_{k}$ and $n_{k} c_{k}^{*}$ is statistically equivalent to $n_{k}$.

The M\&M algorithm is derived from the observation of a few consecutive samples $\{z(k)\}$.

But in NDA mode, we cannot remove the dependence of $x(k)$ on $c_{k}$ in the above way since $c_{k}$ is unknown. In MPSK modulations, we could rewrite Eq.1 as follows.

$$
x(k)=e^{j\left(2 \pi \Delta f k T+\theta+\theta_{k}\right)}+n_{k}
$$

where $\theta_{k}$ is the argument of $c_{k}$ and it satisfies the following equation for MPSK symbols.

$\theta_{k}=2 \pi l / M, l=0,1, \ldots, M-1$

Eq. 3 could be written in the form:

$$
x(k)=e^{j\left(2 \pi \Delta f k T+\theta+\theta_{k}\right)}\left[1+\tilde{n}_{k}\right]
$$

with $\tilde{n}_{k}=n_{k} e^{-j\left(2 \pi \Delta f k T+\theta+\theta_{k}\right)}=R\left\{\tilde{n}_{k}\right\}+j I\left\{\tilde{n}_{k}\right\}$, where $R\{$.$\} and I\{$.$\} means the real part and$ imaginary part of a complex number respectively.

From Eq.4 we get

$$
\arg [x(k)]=\left[2 \pi \Delta f k T+\theta+\theta_{k}+\arg \left[1+\tilde{n}_{k}\right]\right]_{2 \pi}
$$

When Es/No $>>1$, the Eq. 7 is satisfied.

$$
\arg \left[1+\tilde{n}_{k}\right]=\arg \left[1+R\left\{\tilde{n}_{k}\right\}+j I\left\{\tilde{n}_{k}\right\}\right] \approx I\left\{\tilde{n}_{k}\right\}
$$

Next, substituting into Eq.4 yields

$$
x(k) \approx e^{j\left(2 \pi \Delta f k T+\theta+\theta_{k}+I\left\{\tilde{n}_{k}\right\}\right)}
$$

We could remove the dependence make of $x(k)$ on $\theta_{k}$ or on $c_{k}$ by some nonlinear operations such as expanding the argument of $x(k)$ by $\mathrm{M}$ times.

We get the sequence $\{\tilde{z}(k)\}$ as follows, which is the basis of our frequency estimation algorithm.

$$
\tilde{z}(k)=e^{j\left(M * 2 \pi \Delta f k T+M * \theta+M * \theta_{k}+M * I\left\{\tilde{n}_{k}\right\}\right)}
$$

With the reverse process from Eq.3 to Eq.8, we can rewrite Eq.9 as follows.

$$
\tilde{z}(k) \approx e^{j\left(2 M \pi \Delta k T+M^{*} \theta\right)}+\tilde{\tilde{n}}_{k}
$$

where $\tilde{\tilde{n}}_{k}$ is the approximate equivalent noise from phase $M * I\left\{\tilde{n}_{k}\right\}$.

\section{The New Carrier Frequency Offset Estimation Method}


The original M\&M algorithm is in DA mode and is like Eq.11.

$\Delta \widehat{f}=\frac{1}{2 \pi T} \sum_{m=1}^{N} w(m) \times[\arg \{R(m)\}-\arg \{R(m-1)\}]_{2 \pi}$

where $w(m)$ is the weight coefficient and $R(m)$ is the correlation value of $z(k)$.

$$
\begin{aligned}
& R(m)=\frac{1}{L_{0}-m} \sum_{k=m}^{L_{0}-1} z(k) z^{*}(k-m) \quad 1 \leq m \leq N \\
& w(m) \triangleq \frac{3\left[\left(L_{0}-m\right)\left(L_{0}-m+1\right)-N\left(L_{0}-N\right)\right]}{N\left(4 N^{2}-6 N L_{0}+3 L_{0}^{2}-1\right)}
\end{aligned}
$$

where $L_{o}$ means the number of sequence symbols and $N$ should satisfy that $N<L_{o} / 2$.

Comparing Eq.2 and equation Eq.10, we could derive the NDA M\&M algorithm as follows.

$\widehat{y}=\frac{1}{2 M \pi T} \sum_{m=1}^{N} y(m) \times[\arg \{\tilde{R}(m)\}-\arg \{\tilde{R}(m-1)\}]_{2 \pi}$

Where $\widetilde{R}(m)$ is the correlation value of $\tilde{z}(k)$.

$\tilde{R}(m)=\frac{1}{L_{0}-m} \sum_{k=m}^{L_{0}-1} \tilde{z}(k) \tilde{z}^{*}(k-m) \quad 1 \leq m \leq N$

From Eq.14, we could see that to ensure the estimation value correct, the following equation should be satisfied.

$$
\arg \{\widetilde{R}(m)\}-\arg \{\widetilde{R}(m-1)\} \in[-\pi, \pi]
$$

So we get the normalized frequency offset estimation range as $\Delta f T \in[-1 / 2 M, 1 / 2 M]$.

To reach high estimation accuracy, we should set $L_{0}$ and $\mathrm{N}$ large enough, which causes the algorithm too complex to implement. To reduce the computational complexity, we introduce a step factor into Eq.14 and get the following algorithm.

$$
\left.\widehat{\forall}=\frac{1}{2 M \sigma t} \sum_{m-1, H d,+2 d, \ldots, N} w(m) \times \arg \{\tilde{R}(m)\}-\arg \{\tilde{R}(m-d)\}\right]_{2 \pi}
$$

where $d$ is the step factor which should be less than $N$.

Eq. 17 is the samples from Eq.14 and apparently it is simpler to implement than the earlier one, which is just the reason why we name this method as the S-M\&M algorithm.

When $d$ is small, we could approximate $w(m)$ as follows.

$$
\widetilde{w}(m)=\left\{\begin{array}{l}
w(m), m=k d+1 \\
w(k d+1), m \in[k d+2,(k+1) d]
\end{array}\right.
$$

Replace $w(m)$ with Eq.18 in Eq.14, we get the following equation.

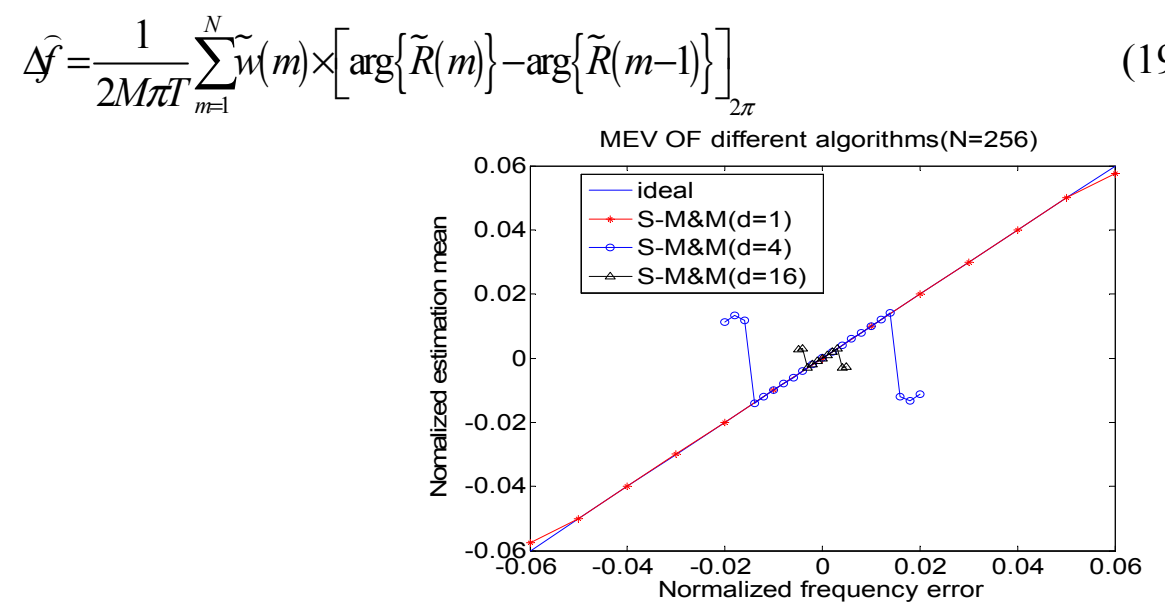

Fig. 2 The estimation average value of different algorithms 
Comparing Eq.19 with Eq.17, we know that if the Eq.20 is satisfied, Eq.17 equals Eq.19 and we can therefore approximate Eq.14 by Eq.17.

$$
[\arg \{\widetilde{R}(m)\}-\arg \{\widetilde{R}(m-d)\}]_{2 \pi}=\sum_{k=m+1-d}^{m}[\arg \{\widetilde{R}(k)\}-\arg \{\widetilde{R}(k-1)\}]_{2 \pi}
$$

To satisfy the Eq.20, we get the following equation.

$$
\arg \{\widetilde{R}(m)\}-\arg \{\widetilde{R}(m-d)\} \in[-\pi, \pi]
$$

Compare it with Eq.16 and apparently we get the conclusion that the S-M\&M algorithm's computational complexity falls at the cost of its estimation range, which makes

$\Delta f T \in[-1 / 2 d M, 1 / 2 d M]$.

Fig. 2 illustrates the estimation average value, $E[\hat{f f T}]$ versus $\Delta f T$. The ideal line is indicated as a reference. We assume the modulation scheme is 8PSK, the estimation interval is of $L_{o}=1024$ symbols and we set the parameter $N$ to be 256. It is apparent that the correct estimation range satisfies $\Delta f T \in[-1 / 2 d M, 1 / 2 d M]$ and it falls as a result of the increase of the step factor $d$.

In next section, we will discuss some important issues including 1) whether this new algorithm is biased or not; and 2) how its performance compares with the M\&M and the Fitz algorithms. Before proceeding we will first introduce the NDA structure of Fitz algorithm.

From the above steps of changing the DA M\&M algorithm into the NDA M\&M algorithm as Eq.14, we could easily get the NDA form of the Fitz algorithm which reads

$$
\Delta \widehat{f}=\frac{1}{M \pi T N(N+1)} \sum_{m=1}^{N} \arg \{\widetilde{R}(m)\}
$$

With Eq.22, the normalized frequency offset estimation range $\Delta f T$ is less than $1 / 2 \mathrm{MN}$.

\section{Computer simulations}

We make some computer simulations to compare the performance of the S-M\&M algorithm with other algorithms.

From Fig.2 we could see that the S-M\&M algorithm is unbiased over the range $|\Delta f T| \leq 1 / 2 d M$.

Fig. 3 compares normalized variance performance for different algorithms. We assume $N$ equals 256 and the normalized frequency offset $|\Delta f T|<=1 / 2 M N$, which is necessary for the Fitz algorithm. The MCRB is also shown as a baseline.

From the figure we can easily get the following three points. 1) All three of the Fitz, M\&M and $\mathrm{S}-\mathrm{M} \& \mathrm{M}$ algorithms approach to the MCRB as the Eb/No increases; 2) Comparing with the M\&M algorithm, the estimation accuracy of the S-M\&M algorithm appears to have little loss as the step factor $d$ increases, which will also reduces the estimation range. So, according to the frequency offset range to be estimated, we have the choice of reducing the computational complexity

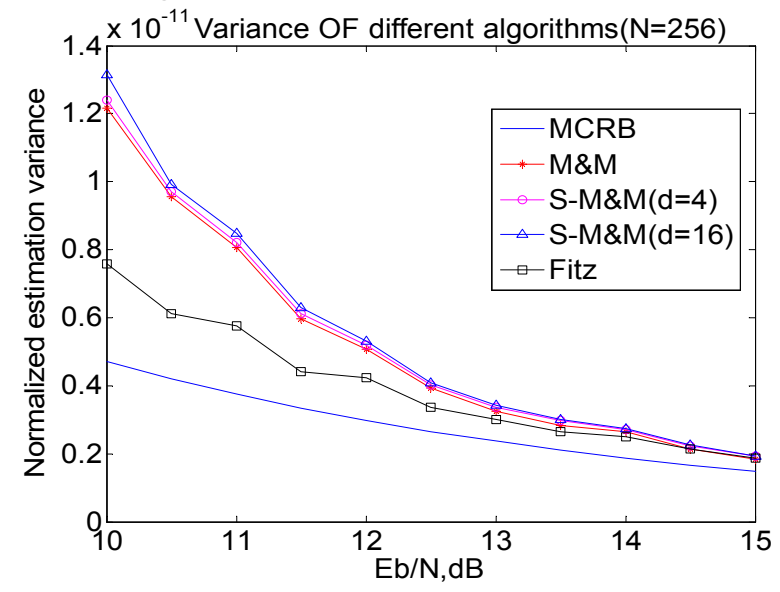

The estimation variance of different algorithms

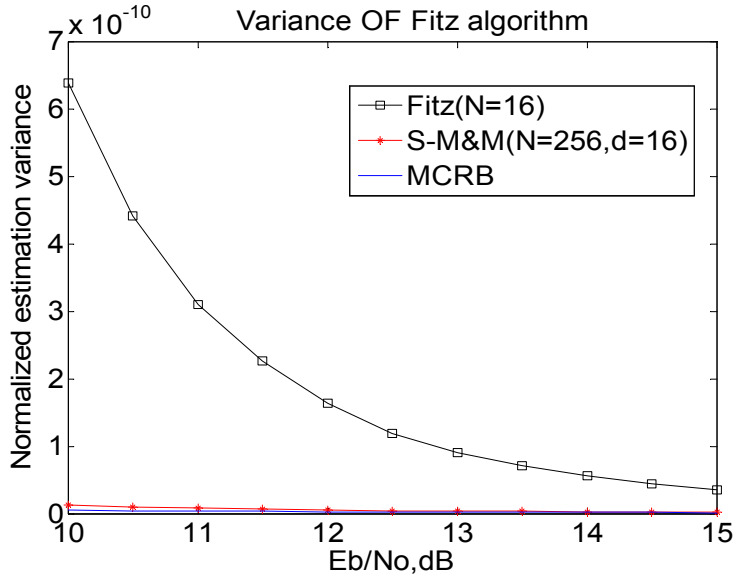

The estimation variance of the Fitz algorithm 
by introducing the step factor $d$ with little loss of estimation accuracy; and 3) Although the NDA Fitz algorithm excels in the estimation accuracy over the S-M\&M algorithm when the parameters $L_{o}$ and $N$ are set the same for both of the algorithms, this makes the estimation range of the S-M\&M algorithm to be $N / d$ times of that of the Fitz algorithm.

Fig. 4 gives out the variance performance comparison of the Fitz and the S-M\&M algorithms when they have the same estimation range. We assume the normalized frequency offset $|\Delta f T|=0.003$. We set $N$ to be 16 for the Fitz algorithm and set $N$ to be 256 and $d$ to be 16 for the S-M\&M algorithm, so the two algorithms have the same estimation range $|\Delta f T| \leq 1 / 256$. From the figure we could easily know that the estimation accuracy of the S-M\&M algorithm is much better than that of the Fitz algorithm. We can analyze the reason as follows. The Fitz algorithm controls the estimation range and estimation accuracy by the same parameter $N$, and we can only increase the estimation range at the expense of worse estimation accuracy, or improve the estimation accuracy at the cost of reduced estimation range. But it is not the case for the S-M\&M algorithm, which controls the estimation accuracy and range by parameter $N$ and $d$ separately. So the S-M\&M algorithm is more flexible than the Fitz algorithm and can have larger estimation range with the same estimation accuracy.

\section{Conclusion}

We have proposed a new non-data-aided algorithm of carrier frequency estimation for MPSK modulations. It is a simplified version of the M\&M algorithm and we name it the S-M\&M algorithm.

When the Fitz algorithm encounters the contradiction of estimation range and estimation accuracy, the S-M\&M algorithm resolves the problem as the M\&M algorithm does. So taking the estimation range and estimation accuracy into account synthetically, we can draw a conclusion that the S-M\&M outperforms the Fitz algorithm.

By introducing the step factor $d$ into the M\&M algorithm, the S-M\&M algorithm can reduce the computational complexity without apparent loss in the estimation accuracy. We can easily see that the S-M\&M algorithm is also the generalization of the M\&M algorithm and the two algorithms equal when $d$ is set to be 1 . So we can say that the S-M\&M algorithm is more flexible than the M\&M algorithm in some sense that we have the choice of reducing the computational complexity with little loss of the estimation accuracy according to the frequency offset range to be estimated.

\section{References}

[1] H. Meyr, M. Moeneclaey, and S. Fetchel: Digital Communication Receivers: Synchronization, Channel Estimation, and Signal Processing. New York: Wiley (1997).

[2] Yimin Jiang, Feng-Wen Sun and Baras. J. S; Carrier and Bit Synchronization in Data Communication--A Tutorial Review. IEEE Transactions on Information Theory; vol.49 (2003), p.191-203.

[3] Fusco. T, Petrella. A and Tanda. M: Data-aided symbol timing and CFO synchronization for filter bank multicarrier systems. IEEE Transactions on Wireless Communications; vol.8 (2009), p. 2705-2715.

[4] M. P. Fitz: Planar filtered techniques for burst mode carrier synchronization. Proc. IEEE GLOBECOM'91, Phoenix, AZ; (1991).

[5] M. Luise and R. Reggiannini: Carrier frequency recovery in all-digital modems for burst-mode transmissions. IEEE Trans. Commun; vol. 43 (1995), p.1169-1178.

[6] Umberto Mengali and M. Morelli: Data-Aided Frequency Estimation for Burst Digital Transmission. IEEE Trans. Commun; vol. 45 (1997), p.23-25. 